\title{
No Correlations Between the Development of Specific IgA and IgM Antibodies Against Anti-TNF Blocking Agents, Disease Activity and Adverse Side Reactions in Patients with Rheumatoid Arthritis
}

\author{
Maurizio Benucci ${ }^{*}, 1$, Gianantonio Saviola ${ }^{2}$, Francesca Meacci ${ }^{3}$, Mariangela Manfredi ${ }^{3}$, \\ Maria Infantino ${ }^{3}$, Paolo Campi ${ }^{4}$, Maurizio Severino ${ }^{4}$, Miriam Iorno ${ }^{4}$, \\ Piercarlo Sarzi-Puttini ${ }^{5}$ and Fabiola Atzeni ${ }^{5}$
}

\author{
${ }^{1}$ Rheumatology Unit, Nuovo Ospedale S. Giovanni di Dio, ASL 10, Florence, Italy \\ ${ }^{2}$ Rheumatology and Rehabilitation Unit IRCCS S. Maugeri, Mantua, Italy \\ ${ }^{3}$ Immunology and Allergology Laboratory Unit, Nuovo Ospedale S. Giovanni di Dio, ASL 10, Florence, Italy \\ ${ }^{4}$ Allergy and Clinical Immunology Unit, Nuovo Ospedale S. Giovanni di Dio, ASL 10, Florence, Italy \\ ${ }^{5}$ Rheumatology Unit, L. Sacco University Hospital, Milan, Italy
}

\begin{abstract}
The use of tumour necrosis factor (TNF) antagonists (infliximab [IFN], etanercept [ETN], adalimumab [ADA]) has changed the course of many rheumatic diseases, including rheumatoid arthritis (RA). However, some questions concerning their safety have emerged since their approval because they can trigger immunisation, induce rare type I and III hypersensitivity, and cause acute and delayed reactions. The aim of this study was to evaluate the correlations between hypersensitivity reactions to biological agents, disease activity and the development of class-specific IgA and IgM antibodies against the three anti-TNF agents in patients with RA.

This longitudinal observational study involved consecutive outpatients with active RA who started treatment with IFN $(n=30)$, ETN $(n=41)$ or ADA $(n=28)$. Clinical data and systemic and local side effects were collected prospectively at baseline and after six months of anti-TNF treatment. Serum samples were taken at the same time points in order to measure antibodies against the TNF blockers, anti-nuclear (ANA) and anti-dsDNA antibodies. The IgA and IgM antibodies specific to all three anti-TNF- $\alpha$ agents were analysed using ImmunoCaP Phadia- Thermofisher especially developed in collaboration with the laboratory of Immunology and Allergy, San Giovanni di Dio, Florence.

The mean age of the 99 patients ( $86 \%$ females) was $54.6 \pm 12.4$ years, and the median disease duration was 11.2 \pm .3 .2 years (range 3-14.3). The three treatment groups were comparable in terms of age, gender, rheumatoid factor and anticitrullinated peptide (CCP) antibody positivity, and baseline C-reactive protein levels, erythrocyte sedimentation rate, 28joint disease activity scores, and concomitant medications. Twelve patients treated with INF (40\%) had anti-IFN IgM, and two $(6 \%)$ anti-IFN IgA; 19 patients treated with ADA (68\%) had anti-ADA IgM, and four (6\%) anti-ADA IgA; and 27 patients treated with ETN (66\%) had anti-ETN IgM, and $24(58 \%)$ anti-ETN IgA. There were five systemic reactions in the IFN group, and seven adverse local reactions in both the ADA and the ETN group. There was no correlation between drug-specific $\operatorname{Ig} \mathrm{A}$ and $\operatorname{IgM}$ antibodies $(\mathrm{p}=0.65)$. There was also no correlation between the antibodies and disease activity after six months of treatment $(\mathrm{r}=0.189 ; \mathrm{p}=0.32)$.

Our findings show that the development of antibodies against IFN, ADA or ETN of IgA and IgM class are not related to any decrease in efficacy or early discontinuation of anti-TNF treatment in RA patients, nor to systemic and local reactions. Further studies of larger series of RA patients are needed to confirm the relationships between the development of drug-specific antibodies, serum TNF blocker levels, and disease activity.
\end{abstract}

Keywords: TNF blockers, antibodies, adverse events, disease activity, rheumatoid arthritis.

\section{INTRODUCTION}

More than 400,000 patients throughout the world have been treated with anti-tumour necrosis factor (TNF) drugs

*Address correspondence to this author at the Rheumatology Unit, Azienda Sanitaria di Firenze, Hospital S. Giovanni di Dio, Via Torregalli 3, 50143 Florence FI, Italy; Tel: +39-55-7192331; Fax: +39-55-7192306;

E-mails: maubenucci@tiscali.it, maurizio.benucci@asf.toscana.it (infliximab [IFN], etanercept [ETN], adalimumab [ADA]) because of rheumatoid arthritis (RA), psoriatic arthritis (PA) and seronegative spondyloarthritis. However, some questions concerning their safety have emerged since their approval because they can trigger immunisation, induce rare type I and III hypersensitivity, and cause acute and delayed reactions [1]. 
There have been many reports of reactions in patients receiving intravenous IFN, a chimeric $\operatorname{IgG} 1 \mathrm{~K}$ anti-TNF agent [2], and immune-mediated side effects such as various cutaneous reactions have been encountered during therapy subcutaneous anti-TNF drugs. One recent paper has described injection site reactions in $29.3 \%$ patients treated with ETN [3]. The adverse reactions to biological agents have been categorised into five types, including a complemented-mediated reaction with immediate $\operatorname{IgE}$ or delayed $\operatorname{IgG}$ antibody formation [4].

Biological therapeutic agents may be recognised by the human immune system as 'non-self' and induce an immune response (also known as immunogenicity). Endogenous therapeutic proteins such as erythropoietin and growth factors have amino acid sequences that are identical to those of the human equivalent and can be immunogenic as a result of glycosylation or conformational changes that expose new epitopes. Therapeutic antibodies carry unique complementarity determining regions that contain stretches of sequences that are frequently recognised as foreign and induce the formation of anti-drug antibodies. This has clinical implications insofar as it can lead to adverse events, the most common of which are infusion reactions associated with a decrease in drug activity. A number of studies have shown that these antibodies play a role in the development of immunogenicity, but only a few have investigated the correlations between the different isotypes of immunoglobulin (Ig) and disease activity.

The aim of this study was to evaluate the correlations between hypersensitivity reactions to biological agents, disease activity and the development of class-specific IgA and IgM antibodies against the three TNF blockers.

\section{METHODS}

We evaluated 99 RA patients (30 treated with IFN, 28 with ADA, and 41 with ETN) with a mean age of 54.6 \pm 12.4 years and a median disease duration of 11.2 \pm .3 .2 years (range 3-14.3). Eighty-six percent were females. The three treatment groups were comparable in terms of age, gender, rheumatoid factor (RF) and anti-citrullinated peptide (CCP) antibody positivity, and baseline C-reactive protein (CRP) levels, erythrocyte sedimentation rate (ESR), 28-joint disease activity scores (DAS-28), and concomitant medications (Table 1).
DAS28 and the number of local and systemic adverse reactions were evaluated at baseline and after six months of treatment, and drug-specific $\operatorname{IgA}$ and $\operatorname{IgM}$ antibodies were measured using ImmunoCaP Phadia- Thermofisher especially developed in collaboration with the laboratory of Immunology and Allergy, San Giovanni di Dio, Florence. The technology of ImmunoCaP is based on an extremely high total binding capacity per mg of cellulose combined with an optimal amount of cellulose in each solid phase, and ensures the binding of all relevant antibodies, regardless of antibody affinity, while still showing low non-specific binding levels. The ImmunoCAP solid phase consists of a cellulose derivative enclosed in a capsule, and the hydrophilic, highly branched polymer provides an ideal microenvironment in which allergens are irreversibly bound while maintaining their native structure. Anti-nuclear antibodies (ANAs) and anti-double-stranded DNA (dsDNA) antibodies were measured at the same times.

\section{Statistical Analysis}

The descriptive data are shown as mean values \pm standard deviations (SD), and were compared using Student's $t$ test. The correlations were assessed by means of Pearson's correlation coefficient. All of the tests were twosided and a $\mathrm{P}$ value of $<0.05$ was considered statistically significant. The analyses were made using SPSS statistical software (version 14.0) and StatXact-7 (version 7, Cytel Software Corporation, Cambridge, MA).

\section{RESULTS}

After six months, 12 of the 30 patients treated with IFN (40\%) had anti-IFN IgM, and two (6\%) anti-IFN IgA; 19 of the 28 patients treated with ADA $(68 \%)$ had anti-ADA IgM, and four $(6 \%)$ anti-ADA $\operatorname{IgA}$; and 27 of the 41 patients treated with ETN (66\%) had anti-ETN IgM, and $24(58 \%)$ anti-ETN IgA.

There were five systemic reactions in the IFN group, and seven adverse local reactions in both the ADA and the ETN group. There was no correlation between the drug-specific $\operatorname{IgA}$ and $\operatorname{IgM}$ antibodies $(\mathrm{p}=0.65)$, and no correlation between drug-specific antibodies and the development of adverse events $(\mathrm{p}=0.99)$.

Table 1. Characteristics of Patients Included in the Study

\begin{tabular}{|c|c|c|c|}
\hline & INFLIXIMAB 30 pts & ADALIMUMAB 28 pt & ETANERCEPT $41 \mathrm{pt}$ \\
\hline Age (yrs SD) & $52.3 \pm 12.2$ & $53.6 \pm 11.8$ & $54.5 \pm 12.9$ \\
\hline Rheumatoid factor positivity & $76 \%$ & $78 \%$ & $75 \%$ \\
\hline Anti-CCP positivity & $81 \%$ & $79 \%$ & $78 \%$ \\
\hline CRP mg/dl & $3.2 \pm 1.7$ & $3.4 \pm 1.5$ & $3.7 \pm 1.4$ \\
\hline DAS28 & $5.46 \pm 2.1$ & $5.43 \pm 1.9$ & $5.38 \pm 1.8$ \\
\hline Corticosteroids dose mg/day & $6.1 \pm 1.2$ & $6.4 \pm 1.1$ & $6.3 \pm 1.2$ \\
\hline Methotrexate dose mg/week & $12.5 \pm 2.2$ & $12.1 \pm 2.1$ & $11.9 \pm 2.4$ \\
\hline
\end{tabular}


There was also no correlation between the antibodies and disease activity after six months of treatment $(\mathrm{r}=0.189$; $\mathrm{p}=0.32)$ (Figs. 1-4).

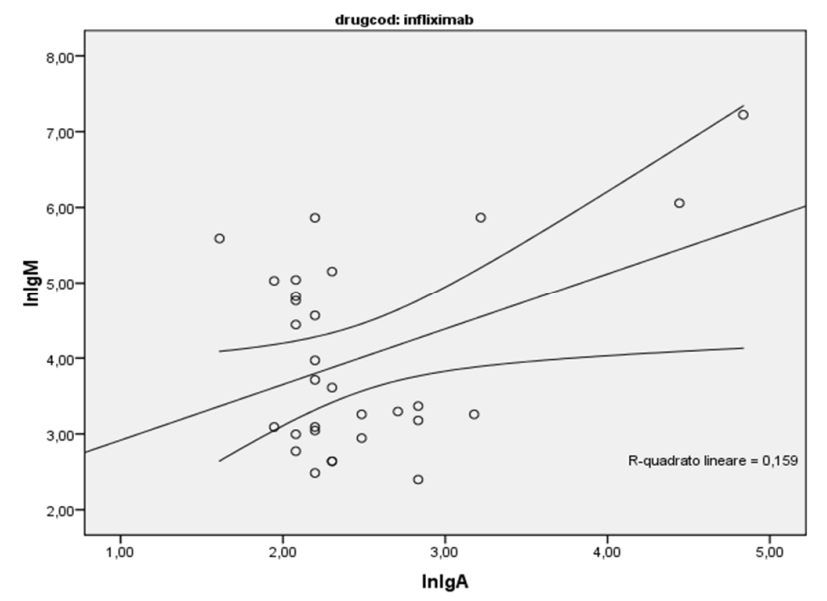

\section{DISCUSSION}

Our findings show that the presence of drug-specific $\operatorname{IgM}$ and IgA antibodies in patients with RA does not correlate

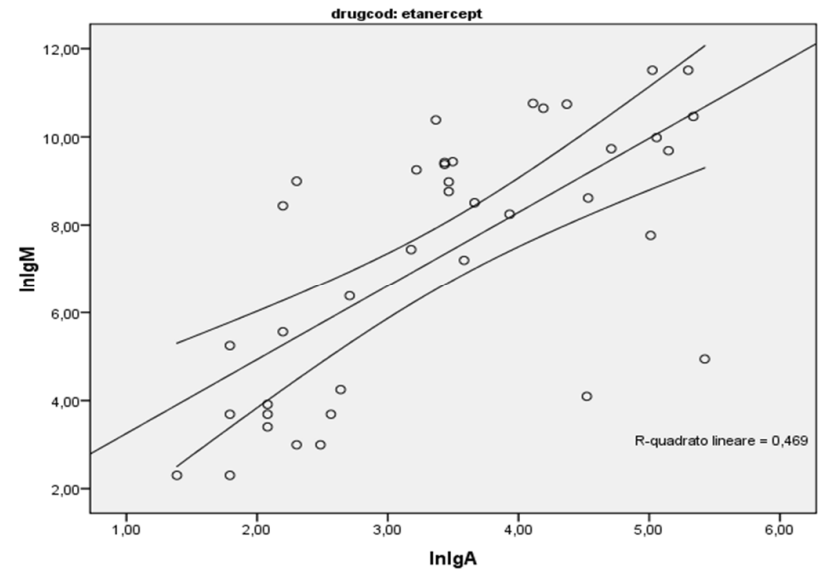

Fig. (1). Correlation between IgA and IgM anti Drugs and Adverse Reactions.
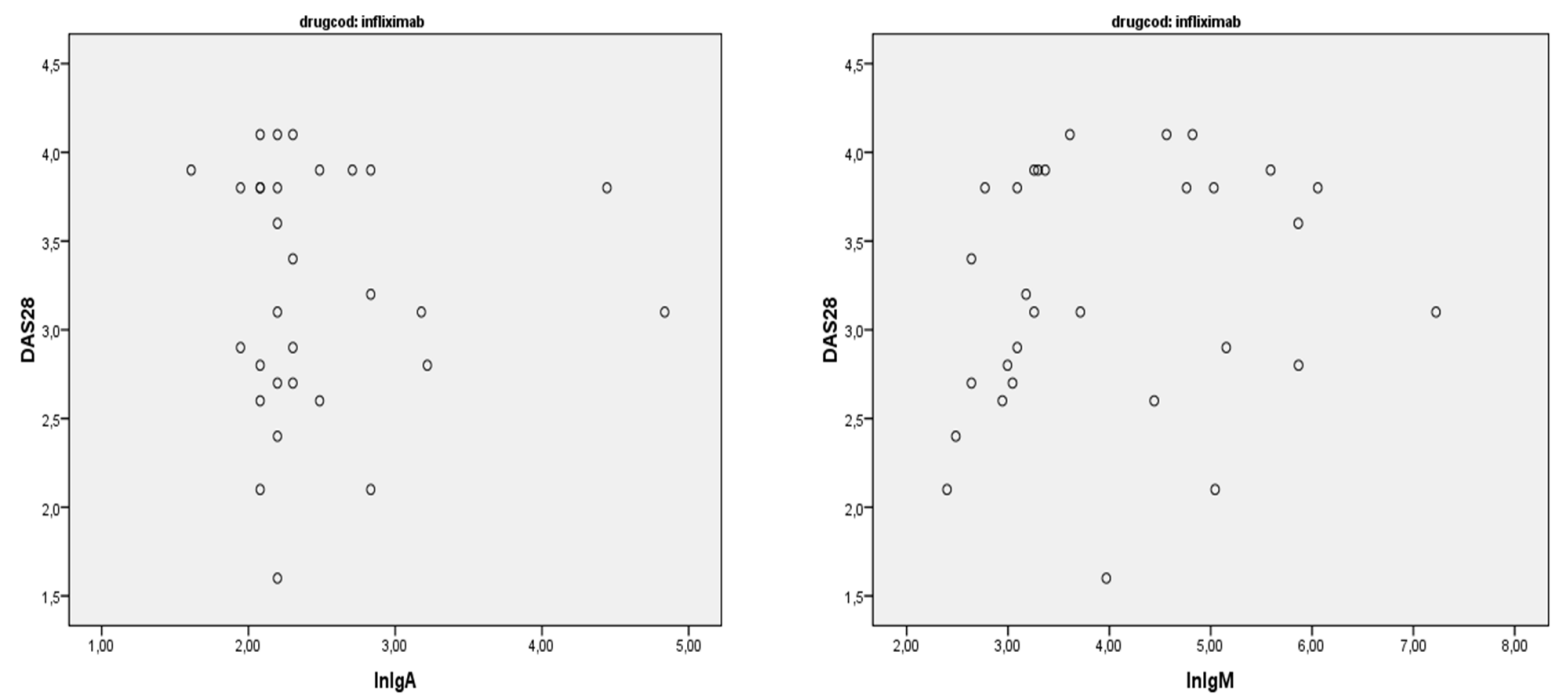

Fig. (2). Correlation between IgA and IgM anti Infliximab and DAS28. 

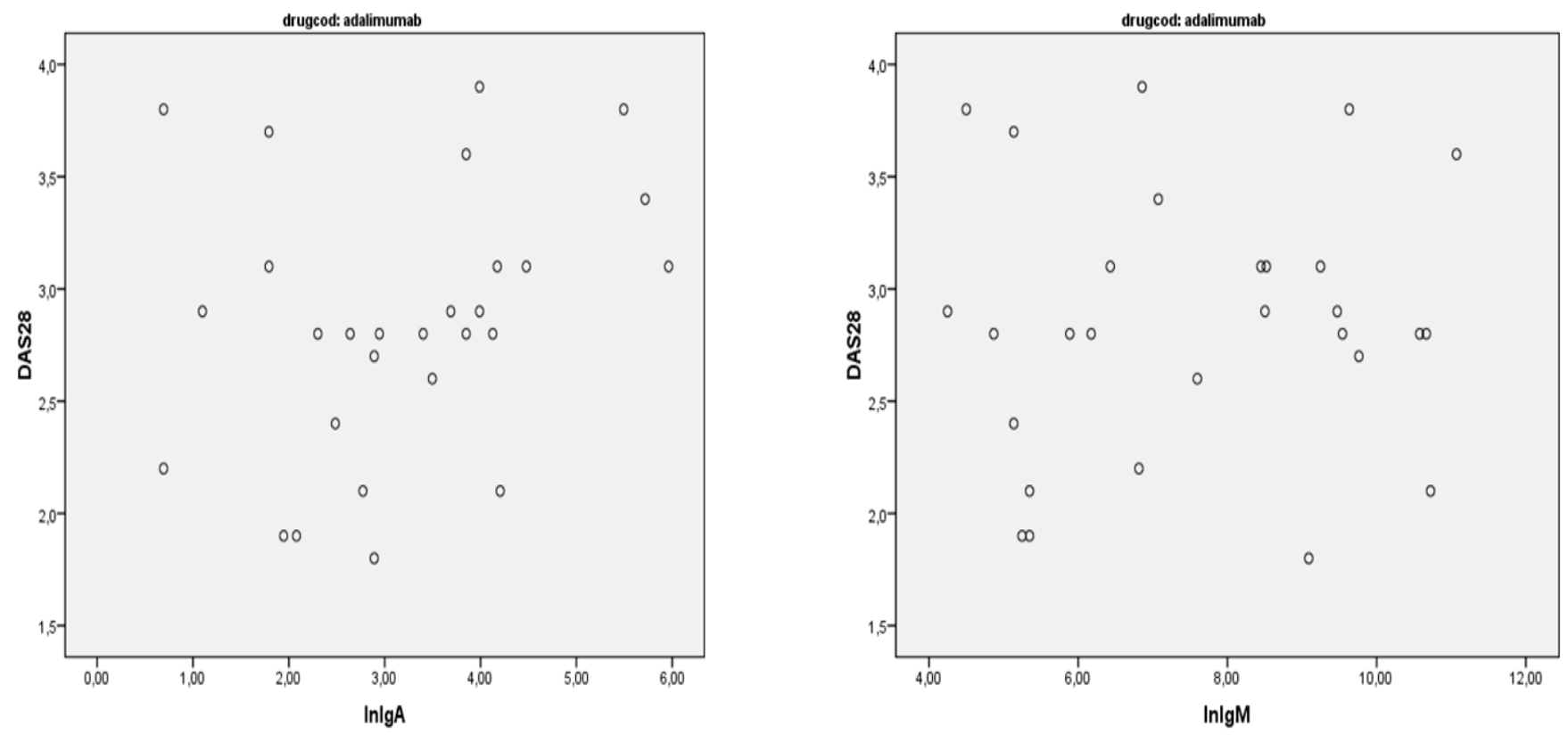

Fig. (3). Correlation between $\operatorname{Ig} \mathrm{A}$ and IgM anti Adalimumab and DAS28.
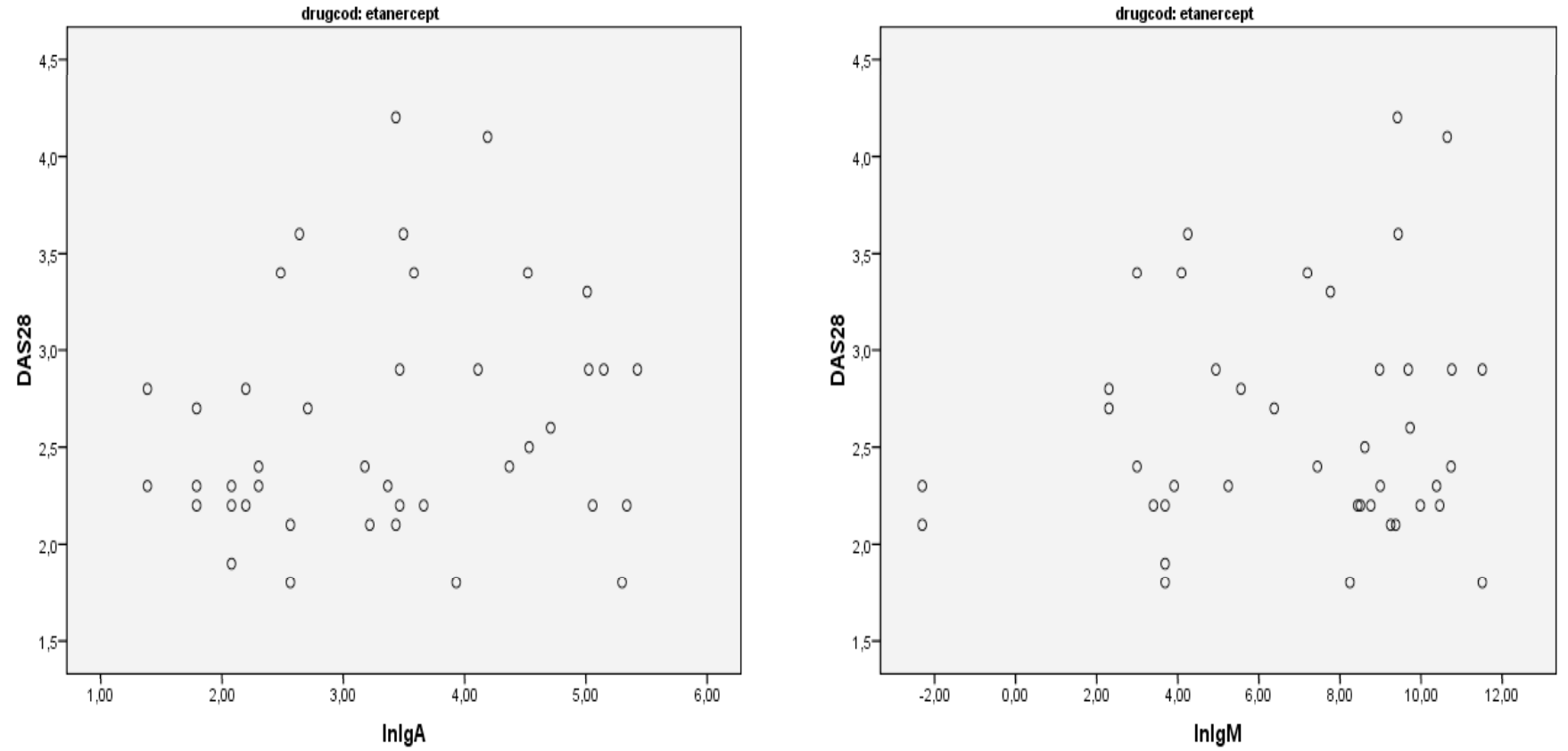

Fig. (4). Correlation between IgA and IgM anti Etanercept and DAS28.

with systemic or local site reactions or disease activity.

Class-specific anti-drug antibodies can be involved in adverse reactions during the treatment of RA, and various findings concerning adverse reactions, disease activity and the development of class- specific anti-drug antibodies have been described. The reactions have been related to IFN immunogenicity and the development of various antibodies.

A paper evaluated the development of $\operatorname{IgE}$ and non- $\operatorname{IgE}$ anti-IFN antibodies, and their relationship with infusion reactions in 71 patients (11 reactive, 11 therapeutic nonresponders, and 49 unreactive therapeutic responders) using the ImmunoCAP assay for IgE and an ELISA assay for IgM and non-isotype-specific anti-IFN antibodies [5]. Eleven of the 71 patients experienced a hypersensitivity reaction to IFN; non-isotype-specific anti-IFN antibodies were detected in eight reactive and two non-responding patients; and three patients with severe reactions had anti-IFN IgE antibodies and positive skin tests. Detectable levels of anti-IFN IgM antibodies were found in three additional patients with negative $\operatorname{IgE}$ and skin test results. Anti-IFN $\operatorname{IgE}$ and $\operatorname{IgM}$ antibodies were not detectable in the two non-responding patients [5]. In another our paper published we didn't observe a correlation between atopy and hypersensitivity reactions during therapy with three different TNF- blocking agents in rheumatoid arthritis [6].

A further study has evaluated disease activity, serum ADA concentrations and anti-ADA antibodies at baseline 
and at eight time points over a period of 156 weeks, and also compared treatment discontinuations, minimal disease activity and clinical remission between the patients with and without anti-ADA antibodies. The results showed that the development of anti-drug antibodies is associated with the negative outcome of ADA treatment in RA patients: the patients with anti-ADA antibodies not only discontinued treatment more often and earlier than those without, but also showed greater disease activity during treatment and only rarely achieved clinical remission. The study also found that two-thirds of the anti-ADA antibody-positive patients developed the antibodies during the first 28 weeks of treatment, and that their presence substantially influenced serum ADA concentrations [7].

Two studies of patients switching TNF inhibitors have shown that the reason for the absence of a response to a first anti-TNF drug has implications on responses to a second inhibitor. In the patients who developed anti-drug antibodies against the first drug (IFN or ADA), the response to the second TNF inhibitor (ADA or ETN) was no different from that of TNF-naive patients, whereas the patients who did not respond to the first TNF inhibitor despite adequate serum drug levels and the absence of anti-drug antibodies had a significantly worse response to the second inhibitor than both the TNF-naive patients and the patients with anti-drug antibodies to their first TNF inhibitor [8,9].

Why do some patients develop an anti-drug antibody response while others do not? It is known that the use of concomitant immunosuppressants is associated with a lower frequency of anti-drug antibodies [10,11] and, in some studies, the presence of anti-drug antibodies has been associated with decreased serum drug levels and a diminished response $[12,13]$. Furthermore, in some cases, the combined presence of anti-drug antibodies and therapeutic monoclonal antibody (TmAb) concentrations leads to the formation of immune complexes, and their continuous presence in serum leads to adverse events. Little is known about the safety of TmAb and anti-TmAb antibody immune complexes, but the presence of antibodies anti-IFN and immune complexes of various sizes may be associated with infusion-related hypersensitivity reactions $[7,14]$.

Various classes of immunoglobulins with different affinities can be produced. Moreover, the presence of antidrug antibodies fluctuates over time; immunological tolerance develops at a certain time during treatment and so the timing of blood sampling can affect the detection of immunogenicity. There are a number of assay formats for the detection of anti-drug antibodies [15], but none of the available assays can detect all of the subclasses or idiotypes. The avidity of IgM antibodies is low, and they are therefore difficult to detect, whereas affinity-matured $\mathrm{IgG} 1$ and $\mathrm{IgG} 4$ antibodies are easier to detect. IgE can also be highly avid, but excess IgG makes it difficult to detect the relatively low amounts of IgE. The most widely used assays include direct enzyme-linked immunosorbent assays (ELISAs), which are straightforward but affected by non-specific binding $[15,16]$. Two-site or bridging assays are sensitive and specific, but do not detect IgG4 antibodies and are susceptible to drug interference, and antigen binding tests (ABTs) are specific and less susceptible to drug interference; however, both radio-immunoassays require a laboratory equipped to deal with radioactivity. A fourth type are cell-based assays which are not used frequently for immunogenicity testing, because they are time consuming, complex and unreliable [17].

Therapeutic antibodies are removed from the circulation similarly or slightly more rapidly than endogenous IgG. The production of anti-drug antibodies can accelerate clearance because of the formation of immune complexes that are taken up by the liver and spleen. Furthermore, drug and antidrug antibody complexes are not biologically active if the anti-drug antibodies are directed to the antigen-binding site, which is usually the only foreign part of humanised or fully human therapeutic antibodies. These two mechanisms decrease drug levels and consequently impair treatment responses [18].

One important limitation of our study is the absence of a record of drug levels. A recent longitudinal, observational cohort of RA patients treated with IFN for at least six months assessed treatment intervals, DAS28, trough IFN levels and anti-IFN antibody levels, and found low (and high) IFN serum trough levels are prevalent, interestingly also in patients with low disease activity. Consecutive measurements of serum trough levels and anti-IFN antibodies are reliable in stable patients [19].

The different types of immunoglobulin ( $\operatorname{Ig} \mathrm{A}$ and $\operatorname{IgM}$ ADA), different clearance due to the formation of immune complexes, and the probable absence of anti-drug antibodies with neutralising effects [20] may explain the lack of a correlation between the development of anti-drug antibodies, systemic or local reactions and disease activity.

In many other papers a different percentage of ADA in different diseases was observed. A recent review showed a different percentage of ADA to Infliximab, Adalimumab, Etanercept in spondiloarthritis, psoriatic arthritis and Crohn's Disease, the value could be influenced also by immunosoppressive therapy such as Methotrexate. Moreover in rheumatoid arthritis patients the detection of ADA is counfounded by the presence of Rheumatoid Factor that can mask the epitopes bound in these assay [21].

\section{CONCLUSIONS}

Our findings indicate that the development IFN, ADA or ETN antibodies of $\operatorname{IgA}$ and $\operatorname{IgM}$ class are not related to a decrease in efficacy or the early discontinuation of anti-TNF treatment in RA patients, and that there is no correlation between systemic and local reactions and antibody formation. Further studies of larger series of RA patients are needed to confirm the relationship between the development of drug-specific antibodies, serum TNF blocker levels, and disease activity.

\section{CONFLICT OF INTEREST}

The authors declare that they have no conflict of interest with respect to the authorship and/or publication of this article.

\section{ACKNOWLEDGEMENTS}

Declared none. 


\section{REFERENCES}

[1] Desai SB, Furst DE. Problems encountered during anti-tumour necrosis factor therapy. Best Pract Res Clin Rheumatol 2006; 20:757-90.

[2] Cheifetz A, Smedley M, Martin S, et al. The incidence and management of infusion reactions to infliximab: a large center experience. Am J Gastroenterol 2003; 98:1315-24.

[3] Dore RK, Mathews S, Schechtman J, et al. The immunogenicity, safety, and efficacy of etanercept liquid administered once weekly in patients with rheumatoid arthritis. Clin Exp Rheumatol 2007; 25:40-6.

[4] Pichler WJ, Campi P. Adverse side effects to biological agents. In Pichler WJ editor. Drug Hypersensitivity. Basel: Karger; 2007: pp.160-74.

[5] Vultaggio A, Matucci A, Nencini F, et al. Anti-infliximab IgE and non-IgE antibodies and induction of infusion-related severe anaphylactic reactions. Allergy 2010; 65:657-61.

[6] Benucci M, Manfredi M, Saviola G, Baiardi P, Campi P. Correlation between atopy and hypersensitivity reactions during therapy with three different TNF-alpha blocking agents in rheumatoid arthritis. Clin Exp Rheumatol 2009 ; 27(2):333-6.

[7] Bartelds GM, Krieckaert CL, Nurmohamed MT, et al. Development of antidrug antibodies against adalimumab and association with disease activity and treatment failure during longterm follow-up.JAMA 2011; 305:1460-8.

[8] Bartelds GM, Wijbrandts CA, Nurmohamed MT, et al. Antiinfliximab and anti-adalimumab antibodies in relation to response to adalimumab in infliximab switchers and anti-tumour necrosis factor naive patients: a cohort study. Ann Rheum Dis 2010; 69:817-21.

[9] Jamnitski A, Bartelds GM, Nurmohamed MT, et al. The presence or absence of antibodies to infliximab or adalimumab determines the outcome of switching to etanercept. Ann Rheum Dis 2011; 70:284-8.

[10] Vermeire S, Noman M, Van Assche G, Baert F, D’Haens G, Rutgeerts P. Effectiveness of concomitant immunosuppressive therapy in suppressing the formation of antibodies to infliximab in Crohn's disease. Gut 2007; 56:1226-31.
[11] Krieckaert CL, Bartelds GM, Lems WF, Wolbink GJ. The effect of immunomodulators on the immunogenicity of TNF-blocking therapeutic monoclonal antibodies: a review. Arthritis Res Ther 2010; 12:217.

[12] Radstake TR, Svenson M, Eijsbouts AM, et al. Formation of antibodies against infliximab and adalimumab strongly correlates with functional drug levels and clinical responses in rheumatoid arthritis. Ann Rheum Dis 2009; 68:1739-45.

[13] Wolbink GJ, Vis M, Lems W, et al. Development of antiinfliximab antibodies and relationship to clinical response in patients with rheumatoid arthritis. Arthritis Rheum 2006; 54:711-5.

[14] van der Laken CJ, Voskuyl AE, Roos JC, et al. Imaging and serum analysis of immune complex formation of radiolabelled infliximab and anti-infliximab in responders and non-responders to therapy for rheumatoid arthritis. Ann Rheum Dis 2007; 66:253-6.

[15] Wolbink GJ, Aarden LA, Dijkmans BA. Dealing with immunogenicity of biologicals: assessment and clinical relevance. Curr Opin Rheumatol 2009; 21:211-5.

[16] Hart MH, de Vrieze H, Wouters D, et al. Differential effect of drug interference in immunogenicity assays. J Immunol Methods 2011; 372:196-203.

[17] Wadhwa M, Thorpe R. Unwanted immunogenicity: lessons learned and future challenges. Bioanalysis 2010; 2:1073-84.

[18] Krieckaert C, Rispens T, Wolbink G. Immunogenicity of biological therapeutics: from assay to patient. Curr Opin Rheumatol 2012; 24:306-11

[19] van der Maas A, van den Bemt BJ, Wolbink GJ, et al. Low infliximab serum trough levels and anti-infliximab antibodies are prevalent in rheumatoid arthritis patients treated with infliximab in daily clinical practice: results of an observational cohort study. BMC Musculoskelet Disord 2012; 13:184.

[20] van Schouwenburg PA, Rispens T, Wolbink GJ. Immunogenicity of anti-TNF biologic therapies for rheumatoid arthritis.Nat Rev Rheumatol 2013; 9:164-72.

[21] Vincent FB, Morand EF, Murphy K, Mackay F, Mariette X, Marcelli C. Antidrug antibodies (ADAb) to tumour necrosis factor (TNF)-specific neutralising agents in chronic inflammatory diseases: a real issue, a clinical perspective. Ann Rheum Dis 2013; 72(2):165-78. 